\title{
Protracted Symptoms after Heat Stroke. Is Vasopressin the Culprit?
}

\author{
Thomas Brown* \\ Nova Southeastern University, USA \\ *Corresponding author: Thomas Brown, Nova Southeastern University, 3200 South University Dr. Suite 4372 Fort Lauderdale, Fl. \\ 33328, USA \\ To Cite This Article: Thomas Brown. Protracted Symptoms after Heat Stroke. Is Vasopressin the Culprit?. Am J Biomed Sci \& Res. 2019 - 4(5). \\ AJBSR.MS.ID.000830. DOI: 10.34297/AJBSR.2019.04.000830
}

Received: 眥 August 12, 2019; Published: 眥 August 21, 2019

\begin{abstract}
Nausea is a common finding in patients diagnosed with heat related disorders but there are no reported cases of severe persistent nausea after initial symptoms of heat stroke have resolved. I present a case of a Cross Country athlete that developed protracted nausea, headache and hypertension after experiencing exertional heat stroke.
\end{abstract}

\section{Case Presentation}

The patient is an adolescent male cross-country runner that collapsed at the 5-mile mark during a practice run. At the time he was averaging 7:30 minutes per mile, which was a normal pace for him, although he did report that he felt unusually fatigued at that point. He was acclimatized to running in very hot ambient temperatures as he trained daily at his home in South Central California. Thirty days prior to the event he underwent a surgical procedure to extract his molars and then subsequently a week later developed mononucleosis. Both of these factors limited him from training prior to the event in August 2019.

The patient's initial temperature at the scene reported by EMS was 105 o F. During transport and in the Emergency Department (ED) he experienced 3 generalized seizures and was subsequently intubated to protect his airway. The patient was aggressively cooled with ice packs to the neck, axilla and groin and was hydrated with normal saline and lactated ringers. At admission he was noted to have evidence of significant muscle and liver breakdown, electrolyte abnormalities, leukocytosis, acute renal failure, lactic acidosis and various cardiac dysrhythmias.

\section{Hospital Course}

The patient was stabilized in the ED and was transferred to the Intensive Care Unit (ICU) where he spent 4 days and then 2 days in the step-down unit before being discharged on 8/18/19. The patient recovered uneventfully. In accordance with accepted practice guidelines for heat stroke, the decision was made to discharge the patient. At the time of discharge his abnormal lab values had returned to normal or were trending down and subjectively the patient reported he felt well.

\section{Post Hospitalization Course}

After being discharged the patient began experiencing a decrease in his appetite and then acutely began to experience significant levels of nausea on 8/31/19 which was 2 weeks after discharge. He was unable to tolerate being in any area that was in close proximity to food and eventually resorted to wearing nose plugs to avoid smells. The nausea was associated with multiple episodes of retching, although he only reported frank vomiting on three occasions. He was treated with Compazine and then Zofran, both of which only provided minimal relief. The nausea continued for 6 weeks before resolution. One week after the onset of nausea he also began experiencing headaches which were constant in nature and hypertension. On physical exam his blood pressures were as high as 150 systolic and 86 diastolic. The patient had no prior history of hypertension; his blood pressure on his pre-participation physical exam was 120/76. The headaches and elevated blood pressure readings continued to trend down to normal over a period of 10 weeks after the heat stroke event.

\section{Discussion}

It is not uncommon for patients to experience a multitude of symptoms after an episode of heat stroke. In fact, as many as one third of victims will have serious sequelae including organ failure, 
severe neurological damage and death may even occur in $3-5 \%$ of exertional heat stroke cases [1-3].

The patient's history of recent viral infection associated with mononucleosis should be ascertained as a vital factor in the development of heat stroke and subsequent systemic inflammatory response syndrome (SIRS) in this case [1].

Laboratory studies in humans suggest that concurrent or preexisting inflammatory or infectious conditions are associated with increased risk for heat stroke even in patients that are well acclimatized as was this patient [1]. In addition, there is evidence that the inflammation associated with certain viral infections result in elevated levels of antidiuretic hormone (ADH) [4].

\section{Pathophysiology}

The progression of heat stroke to organ failure is the consequence of direct cytotoxicity of heat and also the downstream signaling events that initiate a complex cascade of inflammatory pathways that have been suspected as the mediators of multi-organ dysfunction $[1,5]$. This cascade results in the development of the systemic inflammatory response syndrome (SIRS). This syndrome results in a complex interaction between immune, autonomic and neuroendocrine systems which may result in manifestations of heat related pathology persisting long after the initial event $[1,6,7]$.

The immune system signals the presence of insults to the brain through different pathways, leading to stimulation of the circumventricular organs and the release of cytokines and neurotoxic mediators [8]. These afferent signals trigger efferent CNS responses leading to the activation of the autonomic nervous system and importantly the hypothalamic-pituitary-adrenal (HPA) axis [7]. Pro-inflammatory cytokines such as Interleukin (IL) 1B and IL-6 are known to be involved in the secretion of vasopressin via stimulation of the HPA axis (Figure 1) [1,4,6,7]. The HPA axis is the main neuroendocrine structure involved in modulating the adaptive response to different stressors through the interconnection of the sympathoadrenal and neurohypophyseal systems. These systems are responsible for catecholamine secretion and vasopressin release respectively [7]. Presently there are limitations to our understanding of the action of cytokines and currently there are few centers that are ordering these levels in heat related disorders.

\section{Heat Stroke

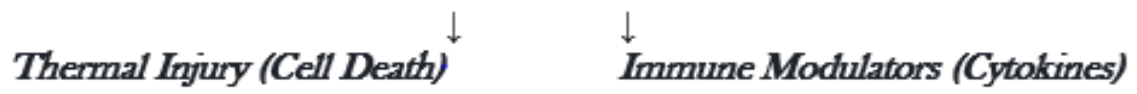 $\downarrow \quad \downarrow$ Systemic Inflammatory Response \\ Organ Failure}

\section{Figure 1}

Vasopressin is a potent vasoconstrictor that is often found to be elevated in SIRS. Elevated levels are associated with nausea, headache and hypertension [9]. Clinically when we think of vasopressin elevation, the disease process of the syndrome of inappropriate antidiuretic hormone (SIADH) comes to mind. That condition is associated with hyponatremia and serum hyposmolarity with high urinary sodium excretion from excessively high levels of vasopressin. I believe in this case that the levels were more consistent with levels achieved when dosing vasopressin therapeutically, such as in the treatment of nocturnal enuresis. The most common side effects from this medication are headache and nausea as is seen in this case.

\section{References}

1. Lisa R Leon, Abderrezak Bouchama (2015) Heat Stoke Comprehensive Physiology. Wiley Online Library 5(2): 611-647.

2. Lew Henry L, Lee Eun Ha, Date Elaine S, Melnik Irina (2002) Rehabilitation of a patient with Heat Stroke: A case report. American Journal of Physical Medicine and Rehabilitation Issue Volume 81(8): 629-632.
3. Abderrezak Bouchama, Mohammed Dehbi, Enrique Chaves Carballo (2007) Cooling and hemodynamic management in heatstroke: practical recommendations. Critical Care 11(3): R54.

4. Se Jin Park, Jae II Shin (2013) Inflammation and hyponatremia: An under recognized condition? Korean Journal of Pediatrics 56(12): 519-522.

5. GM Varghese, G John, K Thomas, OC Abraham, D Mathai (2005) Predictors of multi-organ dysfunction in heatstroke. Emerg Med J 22(3): 185187.

6. Lior Zeller, Victor Novack, Leonid Barski, Alan Jotkowitz, Yaniv Almog (2011) Exertional Heatstroke: clinical characteristics, diagnostics and therapeutic considerations European Journal of Internal Medicine 22(3): 296-299.

7. Valeriu Gheorghita, Alina Elena Barbu, Monica Livia Gheorghiu, Florin Alexandru Caruntu (2015) Endocrine dysfunction in sepsis: a beneficial or deleterious host response? Germs 5(1): 17-25.

8. Jurzak M, Schmid HA (1998) Vasopressin and sensory circumventricular organs. Progress in Brain Research 119: 221-245.

9. Koch Kenneth L Nausea (1991) Vasopressin Lancet 338. 